\title{
Grant-Free Massive Random Access With a Massive MIMO Receiver
}

\author{
Alexander Fengler, Saeid Haghighatshoar, Peter Jung, Giuseppe Caire \\ Communications and Information Theory Group, \\ Technische Universität Berlin \\ E-mail: \{fengler, saeid.haghighatshoar, peter.jung, caire\}@tu-berlin.de.
}

\begin{abstract}
We consider the problem of unsourced random access (U-RA), a grant-free uncoordinated form of random access, in a wireless channel with a massive MIMO base station equipped with a large number $M$ of antennas and a large number of wireless single-antenna devices (users). We consider a block fading channel model where the $M$-dimensional channel vector of each user remains constant over a coherence block containing $L$ signal dimensions in time-frequency. In the considered setting, the number of potential users $K_{\text {tot }}$ is much larger than $L$ but at each time slot only $K_{a} \ll K_{\text {tot }}$ of them are active. Previous results, based on compressed sensing, require that $K_{a} \leq L$, which is a bottleneck in massive deployment scenarios such as Internet-of-Things and U-RA. In the context of activity detection it is known that such a limitation can be overcome when the number of base station antennas $M$ is sufficiently large and a covariance based recovery algorithm is employed at the receiver. We show that, in the context of U-RA, the same concept allows to achieve high spectral efficiencies in the order of $\mathcal{O}(L \log L)$, although at an exponentially growing complexity. We show also that a concatenated coding scheme can be used to reduce the complexity to an acceptable level while still achieving total spectral efficiencies in the order of $\mathcal{O}(L / \log L)$.
\end{abstract}

Index Terms-Random Access (RA), Internet of Things (IoT), Massive MIMO, Grant-Free RA, Unsourced RA.

\section{INTRODUCTION}

One of the paradigms of modern machine-type communications [1] consists of a very large number of devices (here referred to as "users") with sporadic data. Typical examples thereof are Internet-of-Things (IoT) applications, wireless sensors deployed to monitor smart infrastructure, and wearable biomedical devices [2]. In such scenarios, a Base Station (BS) should be able to collect data from a large number of devices. However, due to the sporadic nature of the data generation and communication, allocating some dedicated transmission resource to all users in the system may be extremely wasteful, especially for short messages. On a high level, we distinguish between grant-based and grant-free approaches. In a grantbased protocol the active users are identified and the BS can then allocate transmission resources to the active users, while in a grant-free protocol the users transmit their data right away without awaiting the grant approval of the BS. Interestingly, virtually any exiting cellular standard in operation today $(3 \mathrm{G}$, 4G-LTE, 5G New Radio) makes use of a dedicated random access channel (or slot), followed by some scheduling and resource allocation for the active users [3, 4], therefore these schemes can be seen as examples of grant-based protocols. In contrast, unsourced random access (U-RA) is a novel grant-free paradigm proposed in [5] and motivated by an IoT scenario where millions of cheap devices have their codebook hardwired at the moment of production, and are then disseminated into the environment. In this case, all users make use of the very same codebook and the BS must decode the list of transmitted messages irrespectively of the identity of of the active users. 1

The author of [5] introduced the U-RA model on the real adder multiple-access channel (MAC) with additive white Gaussian noise (AWGN) and established quite tight achievability and converse bounds to the minimum energy per bit over $N_{0}$ required for reliable communications. It was shown that classical MAC schemes like TIN or Aloha perform poorly compared to the random coding achievability bound. Subsequent works introduced many practical coding schemes [6-12], which successively decreased the gap to the achievability bound. In this line of work [8] proposed a coding scheme based on partitioning the transmission slot into subslots, and letting each active user send a codeword from a common codebook across the subslots. The common codebook is obtained by concatenating an outer tree code with an inner compressed sensing code. The inner encoder maps each submessage into one column of a given (real) coding matrix. The inner decoder must identify which columns of the matrix have been transmitted from the received noisy superposition. This is a classical sparse support identification problem, well investigated in the compressed sensing (CS) literature. The inner decoder produces a sequence of activesubmessage-lists across the subblocks. The task of the outer tree decoder is to stitch together the submessages such that each sequence of submessages is a valid path in the tree.

Most of the work on U-RA focused on the real adder MAC with AWGN. Recently the U-RA model was extended to the quasi static fading MAC [13], establishing converse and achievability bounds on $E_{b} / N_{0}$. In this work we extend the U-RA model of [5] to a block-fading MIMO channel, where the channel coefficients remain constant over coherence blocks consisting of $L$ signal dimensions in the timefrequency domain, and change randomly from block to block according to a stationary ergodic process [14]. We refer to

\footnotetext{
${ }^{1}$ If a user wishes to communicate its ID, it can send it as part of the payload. Therefore, in the paradigm of U-RA, if the users make use of individually different codebooks, it would be impossible for the BS to know in advance which codebook to decode since the identity of the active users is not known a priori. Hence, in this context it is in fact essential, and not just a matter of implementation costs, that all users utilize the same codebook.
} 
the average power of the active users as large scale fading coefficients (LSFCs), which are assumed to be deterministic but unknown. The LSFCs vary between different users because of varying distances and large scale-effects such as log-normal shadowing. We formulate the U-RA problem as a joint sparse support recovery problem with multiple measurement vectors (MMVs) [15-17] and leverage recent advances in MIMO activity detection (AD) [18].

A fundamental limitation when considering $\mathrm{AD}$ with a single-antenna $\mathrm{BS}$ is that the required signal dimension $L$ to reliably identify a subset of $K_{a}$ active users among a set consisting of $K_{\text {tot }}$ potentially active users scales as $L=O\left(K_{a} \log \left(\frac{K_{\text {tot }}}{K_{a}}\right)\right)$, thus, almost linearly with $K_{a}$. The key to overcome the linear scaling of $L$ with $K_{a}$ consists of considering quadratic measurements, i.e., sample covariance information. This observation was already empirically provided in [19] where a "much better than linear" regime was experimentally observed and conjectured to be achievable using LASSO applied to the sample covariance matrix of the observation. Also in [19] the importance of the Khatri-Rao product [20] for sparse recovery from MMVs was established for the first time. However, only a linear scaling law was proved because the analysis of LASSO based on coherence is too weak.

In [21, 22] the restricted isometry property (RIP) was shown to hold for Kathri-Rao product matrices and the RIP was subsequently used to show that a covariance based non-negative least-squares (NNLS) estimator can identify the activity of up to $K_{a}=O\left(L^{2} / \log ^{2}\left(\frac{K_{\text {tot }}}{K_{a}}\right)\right)$ active users provided that the number of antennas at the BS grows faster than $K_{a}$. Furthermore, in [18, 22] we presented an improved algorithm for $\mathrm{AD}$ based on maximum-likelihood (ML) estimation of the activity pattern as an unknown vector with non-negative components. The resulting likelihood function minimization is a non-convex problem, that can be solved (approximately) by iterative componentwise minimization. While it is not possible to directly analyze this ML approach, a constrained version of the ML scheme that treats the activity pattern as a binary 0-1 vector lends itself to analysis. The constrained ML scheme yields a combinatorial minimization with exponential complexity and therefore is not useful in practice. However, it was shown that the scaling law for successful detection of the activity pattern was the same as found for the NNLS estimator. Therefore it was conjectured that our original lowcomplexity "relaxed" ML algorithm achieves the same scaling law. 2 A novel approach to the analysis of the the relaxed ML estimator based on the general asymptotic Gaussianity of the ML estimators was recently presented in [25]. This analysis substantially corroborates our conjecture and provides extensive numerical evidence that our ML estimator can identify effectively $K_{a} \approx O\left(L^{2}\right)$ (up to logarithmic terms), in the sense that within this regime and for sufficiently large number of antennas $M$ the estimation error is very small with high probability.

It is evident that the $\mathrm{AD}$ problem and the random access

\footnotetext{
${ }^{2}$ The analysis of the constrained ML scheme was also presented in 23 but it was based on a withdrawn RIP result [24], which has been successively fixed in our analysis in [22].
}

problem are related. In fact, one can immediately obtain a random access scheme from an $\mathrm{AD}$ scheme as follows: assign to each user a unique set of pilot signature sequences (codewords), such that a user, when active, will transmit the signature corresponding to its information message. Since the number of pilot signatures is $K_{\text {tot }} \gg K_{a}$, this scheme involves only an expansion of the number of total users from $K_{\text {tot }}$ to $K_{\text {tot }}^{\prime}=K_{\text {tot }} 2^{B}$ where $B$ is the number of per-message information bits. This idea was recently presented in [26], where the MMV-AMP detector of [17, 27, 28] was used at the receiver side. While conceptually simple, this approach has two major drawbacks: 1) even for relatively small information packets (e.g., $B=100$ bits), the dimension of the pilot matrix is too large for practical computational algorithms; 2) each user has a different set of pilot sequences, and therefore the scheme is not compliant with the basic assumption of U-RA, that all users have the same codebook.

In contrast, we show that a U-RA compliant scheme with a covariance based decoder, in theory, an arbitrary small probability of error is achievable at any $E_{b} / N_{0}$ provided that a sufficiently large number of base station antennas is used, and that the sum spectral efficiency can grow as $\mathcal{O}(L \log (L))$. In practice this is not possible since the complexity would grow exponentially. To bypass this we consider a concatenated scheme, build upon the approach of [8], that does not incur in the large dimension problem and is independent of the number of "inactive" users. In our scheme, the message of $B$ bits of each user is split into a sequence of submessages of potentially different lengths. These submessages are encoded via a tree code (the same for each user), such that the encoded blocks have the same length of $J$ bits. Then, each user transmits its sequence of $J$-bits blocks in consecutive slots of $L$ dimensions, using the same $L \times 2^{J}$ coding matrix (where blocks are encoded in the matrix columns). The inner detector uses the ML activity detection scheme and for each slot recovers the set of active columns of the coding matrix. These are passed to the outer tree code, which recovers each user message by "stitching together" the sequence of submessages as valid paths in the code tree. We show that with the concatenated code the sum spectral efficiency can grow as $\mathcal{O}(L / \log L)$. So it is possible to transmit an arbitrary large amount of bits per signal dimension, provided that the number of antennas at the BS is large enough. This can be achieved in a completely non-coherent way, i.e. it is at no point necessary to estimate the channel matrix (small-scale fading coefficients). These properties make the presented scheme well suited for easy deployable, low-latency, energy efficient communication in an IoT setting.

\section{SYSTEM MODEL}

We consider a block-fading channel with blocks of $L$ signal dimensions over which the user channel vectors are constant. We assume $n=S L$, for some integer $S$, such that the transmission of a codeword spans $S$ fading blocks. Following the problem formulation in [5], each user is given the same codebook $\mathcal{C}=\left\{\mathbf{c}(m): m \in\left[2^{n R}\right]\right\}$, formed by $2^{n R}$ code- 
words $\mathbf{c}(m) \in \mathbb{C}^{n}$. An unknown number $K_{a}$ out of $K_{\text {tot }}$ total users transmits their message over the coherence block. ${ }^{3}$ Let $\mathcal{K}_{a}$ denote the set of active users. The BS must then produce a list $\mathcal{L}$ of the transmitted messages $\left\{m_{k}: k \in \mathcal{K}_{a}\right\}$ (i.e., the messages of the active users). The system performance is expressed in terms of the Per-User Probability of Misdetection, defined as the average fraction of transmitted messages not contained in the list, i.e.,

$$
p_{m d}=\frac{1}{K_{a}} \sum_{k \in \mathcal{K}_{a}} \mathbb{P}\left(m_{k} \notin \mathcal{L}\right),
$$

and the Probability of False-Alarm, defined as the average fraction of decoded messages that were indeed not sent, i.e.,

$$
p_{f a}=\frac{\left|\mathcal{L} \backslash\left\{m_{k}: k \in \mathcal{K}_{a}\right\}\right|}{|\mathcal{L}|} .
$$

The size of the list is also an outcome of the decoding algorithm, and therefore it is a random variable. Notice that in this problem formulation the number of total users $K_{\text {tot }}$ is completely irrelevant, as long as it is much larger than the range of possible active user set sizes $K_{a}$ (e.g., we may consider $K_{\text {tot }}=\infty$ ). Letting the average energy per symbol of the codebook $\mathcal{C}$ be denoted by $E_{s}=\frac{1}{n 2^{n R}} \sum_{m=1}^{2^{n R}}\|\mathbf{c}(m)\|_{2}^{2}$, the received signal can be re-normalized such that the AWGN per-component variance is $\sigma^{2}=N_{0} / E_{s}$ and the received energy per code symbol is 1 . Furthermore, as customary in coded systems, we express energy efficiency in terms of the standard quantity $E_{b} / N_{0}:=\frac{E_{s}}{R N_{0}}$.

For now assume $S=1$, i.e. each user transmits his codeword in a single block of length $L$. Further fix $J=L R$ and let $\mathbf{A} \in \mathbb{C}^{L \times 2^{J}}=\left[\mathbf{a}_{1}, \ldots, \mathbf{a}_{2 J}^{J}\right]$, be a matrix with columns normalized such that $\left\|\mathbf{a}_{i}\right\|_{2}^{2}=L$. Each column of $\mathbf{A}$ represents one codeword. Let $i_{k}$ denote the $J$-bit messages produced by the active users $k \in \mathcal{K}_{a}$, represented as integers in $\left[1: 2^{J}\right]$, user $k$ simply sends the column $\mathbf{a}_{i_{k}}$ of the coding matrix $\mathbf{A}$. The received signal at the $M$-antennas BS takes on the form

$$
\begin{aligned}
\mathbf{Y} & =\sum_{k \in \mathcal{K}_{a}} \sqrt{g_{k}} \mathbf{a}_{i_{k}} \mathbf{h}_{k}^{\top}+\mathbf{Z} \\
& =\mathbf{A} \boldsymbol{\Phi} \mathbf{G}^{1 / 2} \mathbf{H}+\mathbf{Z}
\end{aligned}
$$

where $\mathbf{G}=\operatorname{diag}\left(g_{1}, \ldots, g_{K_{\text {tot }}}\right) \in \mathbb{R}^{K_{\text {tot }} \times K_{\text {tot }} \text { is the diagonal }}$ matrix of deterministic but unknown LSFCs, $\mathbf{H} \in \mathbb{C}^{K_{\text {tot }} \times M}$ is the matrix containing, by rows, the user channel vectors $\mathbf{h}_{k}$ formed by the small-scale fading antenna coefficients (Gaussian i.i.d. entries $\sim \mathcal{C N}(0,1)$ ), $\mathbf{Z} \in \mathbb{C}^{L \times M}$ is the matrix of AWGN samples (i.i.d. entries $\sim \mathcal{C N}\left(0, \sigma^{2}\right)$ ), and $\boldsymbol{\Phi} \in\{0,1\}^{2^{J} \times K_{\text {tot }}}$ is a binary selection matrix where for each $k \in \mathcal{K}_{a}$ the corresponding column $\boldsymbol{\Phi}_{:, k}$ is all-zero but a single one in position $i_{k}$, and for all $k \in \mathcal{K}_{\text {tot }} \backslash \mathcal{K}_{a}$ the corresponding column $\boldsymbol{\Phi}_{:, k}$ contains all zeros.

In line with the classical massive MIMO setting [29], we assume for simplicity an independent Rayleigh fading model, such that the channel vectors $\left\{\mathbf{h}_{k}: k \in \mathcal{K}_{\text {tot }}\right\}$ are independent

\footnotetext{
${ }^{3}$ Here, as in [5] and in [8], we assume that users are synchronized. This assumption is not very restrictive since it is reasonable to assume that all users in the system can listen to a common reference signal.
}

from each other and are spatially white (i.e., uncorrelated along the antennas), that is, $\mathbf{h}_{k} \sim \mathcal{C N}\left(0, \mathbf{I}_{M}\right)$.

Let's focus on the matrix $\mathbf{X}=\mathbf{\Phi} \mathbf{G}^{1 / 2} \mathbf{H}$ of dimension $2^{J} \times$ $M$. The $r$-th row of such matrix is given by

$$
\mathbf{X}_{r,:}=\sum_{k \in \mathcal{K}_{a}} \sqrt{g_{k}} \phi_{r, k} \mathbf{h}_{k}^{\top}
$$

where $\phi_{r, k}$ is the $(r, k)$-th element of $\boldsymbol{\Phi}$, equal to one if $r=$ $i_{k}$ and zero otherwise. It follows that $\mathbf{X}_{r,:}$ is Gaussian with i.i.d. entries $\sim \mathcal{C N}\left(0, \sum_{k \in \mathcal{K}_{a}} g_{k} \phi_{r, k}\right)$. Since the messages are uniformly distributed over $\left[1: 2^{J}\right]$ and statistically independent across the users, the probability that $\mathbf{X}_{r,:}$ is identically zero is given by $\left(1-2^{-J}\right)^{K_{a}}$. Hence, for $2^{J}$ significantly larger than $K_{a}$, the matrix $\mathbf{X}$ is row-sparse.

In order to show the equivalence to the MMV joint-sparsesupport-recovery (JSSR) problem we define the modified LSFC-activity coefficients $\gamma_{r}:=\sum_{k \in \mathcal{K}_{a}} g_{k} \phi_{r, k}$ and $\boldsymbol{\Gamma}=$ $\operatorname{diag}\left(\gamma_{1}, \ldots, \gamma_{2^{J}}\right)$. Then, 3 can be written as

$$
\mathbf{Y}=\mathbf{A} \boldsymbol{\Gamma}^{1 / 2} \widetilde{\mathbf{H}}+\mathbf{Z}
$$

where $\widetilde{\mathbf{H}} \in \mathbb{C}^{2^{J} \times M}$ with i.i.d. elements $\sim \mathcal{C N}(0,1)$. Notice that in (5) the number of total users $K_{\text {tot }}$ plays no role. In fact, none of the matrices involved in (5) depends on $K_{\text {tot }}$.

The task of the decoder at the BS is to identify the nonzero elements of the modified active LSFC pattern $\gamma$, the vector of diagonal coefficients of $\boldsymbol{\Gamma}$. The active (non-zero) elements correspond to the indices of the transmitted messages. Notice that even if two or more users choose the same submessage, the corresponding modified LSFC $\gamma_{r}$ is positive since it corresponds to the sum of the signal powers.

\section{COVARIANCE BASED SUPPORT RECOVERY}

The covariance based approach to the JSSR problem (5) is based on the observation that the columns $\mathbf{y}_{i}$ of $\mathbf{Y}, i=$ $1, \ldots, M$ are Gaussian iid $\sim \mathcal{N}\left(0, \boldsymbol{\Sigma}_{\mathbf{y}}\right)$ with covariance matrix

$$
\boldsymbol{\Sigma}_{\mathbf{y}}=\mathbf{A} \boldsymbol{\Gamma} \mathbf{A}^{\mathrm{H}}+\sigma^{2} \mathbf{I}_{L}
$$

We then attempt to recover $\boldsymbol{\Gamma}=\operatorname{diag}(\gamma)$ from the observed empirical covariance matrix

$$
\widehat{\mathbf{\Sigma}}_{\mathbf{y}}:=\frac{1}{M} \mathbf{Y} \mathbf{Y}^{\mathrm{H}}
$$

For that we consider two estimators, the first is a maximum likelihood (ML) estimator that considers the activity vector $\gamma$ as a deterministic unknown non-negative vector and the second is based on non-negative-least-squares (NNLS). While the former will be used in practice due to its consistently superior performance, the latter is more suitable for giving theoretical achievability guarantees. Both estimators can efficiently be calculated with an iterative componentwise optimization algorithm. For the scaling law analysis of the estimation error if the ML estimator versus the parameters $L, M$ and $K_{a}$, please refer to the comments in Section [1] 


\section{A. Maximum Likelihood}

Let us first consider the Maximum Likelihood (ML) estimator of $\gamma$. We introduce the log-likelihood cost function

$$
\begin{aligned}
f(\gamma) & :=-\frac{1}{M} \log p(\mathbf{Y} \mid \gamma) \stackrel{(a)}{=}-\frac{1}{M} \sum_{i=1}^{M} \log p\left(\mathbf{Y}_{:, i} \mid \gamma\right) \\
& =\log \left|\mathbf{A} \boldsymbol{\Gamma} \mathbf{A}^{\mathrm{H}}+\sigma^{2} \mathbf{I}_{L}\right|+\operatorname{tr}\left(\left(\mathbf{A} \boldsymbol{\Gamma} \mathbf{A}^{\mathrm{H}}+\sigma^{2} \mathbf{I}_{L}\right)^{-1} \widehat{\mathbf{\Sigma}}_{\mathbf{y}}\right)
\end{aligned}
$$

where $(a)$ follows from the fact that the columns of $\mathbf{Y}$ are i.i.d. (due to the spatially white user channel vectors), and where $\widehat{\mathbf{\Sigma}}_{\mathbf{y}}$ denotes the sample covariance matrix of the columns of $\mathbf{Y}$ as in (7). We define the ML estimator as

$$
\gamma_{\mathrm{ML}}^{*}=\underset{\gamma \in \mathbb{R}_{+}^{K_{\text {tot }}}}{\arg \min } f(\gamma) .
$$

Note that the set over that we search for the minimum is larger than the actual non-convex set of permitted signals $\left\{\gamma \in \mathbb{R}_{+}^{K_{\text {tot }}}:\|\gamma\|_{0} \leq K_{a}\right\}$. In that sense (10) is a relaxed version of the actual (constrained) ML estimator. Nonetheless, the constrained ML estimator requires optimization over an exponentially large number of support combinations, which is in general infeasible, while the relaxed version shows to be efficiently computable. Furthermore, 10 does not require any prior knowledge of $K_{a}$.

\section{B. Non-Negative Least Squares}

For

$$
\boldsymbol{\Sigma}(\gamma):=\mathbf{A} \boldsymbol{\Gamma} \mathbf{A}^{\mathrm{H}}+\sigma^{2} \mathbf{I}_{L}
$$

we define the NNLS estimator as

$$
\gamma_{\mathrm{NNLS}}^{*}=\underset{\gamma \in \mathbb{R}_{+}^{K}}{\arg \min }\left\|\boldsymbol{\Sigma}(\gamma)-\widehat{\boldsymbol{\Sigma}}_{\mathbf{y}}\right\|_{\mathrm{F}}^{2} .
$$

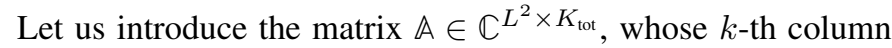
is defined by:

$$
\mathbb{A}_{:, k}:=\operatorname{vec}\left(\mathbf{a}_{k} \mathbf{a}_{k}^{\mathrm{H}}\right) .
$$

and let $\mathbf{w}=\operatorname{vec}\left(\widehat{\boldsymbol{\Sigma}}_{\mathbf{y}}-\sigma^{2} \mathbf{I}_{L}\right)$ denote the $L^{2} \times 1$ vector obtained by stacking the columns of $\widehat{\boldsymbol{\Sigma}}_{\mathbf{y}}-\sigma^{2} \mathbf{I}_{L}$. Then, we can write (12) in the convenient form

$$
\boldsymbol{\gamma}^{*}=\underset{\gamma \in \mathbb{R}_{+}^{K}}{\arg \min }\|\mathbb{A} \boldsymbol{\gamma}-\mathbf{w}\|_{2}^{2}
$$

as a linear least squares problem with non-negativity constraint, known as non-negative least squares (NNLS). Such an algorithm was proposed for the activity detection problem in [30].

NNLS has a special property, as discussed for example in [31] and referred to as the $\mathcal{M}^{+}$-criterion in [32], which makes it particularly suitable for recovering sparse vectors: If the row span of $\mathbb{A}$ intersects the positive orthant, NNLS implicitly also performs $\ell_{1}$-regularization.
Algorithm 1 Joint support recovery via Coordinate-wise Optimization

1: Input: The sample covariance matrix $\widehat{\boldsymbol{\Sigma}}_{\mathbf{y}}=\frac{1}{M} \mathbf{Y} \mathbf{Y}^{\mathrm{H}}$ of the $L \times M$ matrix of samples $\mathbf{Y}$.

2: Input: The LSFCs of $K_{\text {tot }}$ users $\left(g_{1}, \ldots, g_{K_{\text {tot }}}\right)$ if available.

3: Initialize: $\boldsymbol{\Sigma}=\sigma^{2} \mathbf{I}_{L}, \boldsymbol{\gamma}=\mathbf{0}$.

4: for $i=1,2, \ldots$ do

5: $\quad$ Select an index $k \in\left[K_{\text {tot }}\right]$ corresponding to the $k$-th component of $\gamma=\left(\gamma_{1}, \ldots, \gamma_{K_{\text {tot }}}\right)^{\top}$ randomly or according to a specific schedule.

6: $\quad$ ML: Set $d^{*}=\max \left\{\frac{\mathbf{a}_{k}^{H} \boldsymbol{\Sigma}^{-1} \widehat{\boldsymbol{\Sigma}}_{\mathbf{y}} \boldsymbol{\Sigma}^{-1} \mathbf{a}_{k}-\mathbf{a}_{k}^{H} \boldsymbol{\Sigma}^{-1} \mathbf{a}_{k}}{\left(\mathbf{a}_{k}^{H} \boldsymbol{\Sigma}^{-1} \mathbf{a}_{k}\right)^{2}},-\gamma_{k}\right\}$.

7: $\quad$ NNLS: $\operatorname{Set} d^{*}=\max \left\{\frac{\mathbf{a}_{k}^{H}\left(\widehat{\boldsymbol{\Sigma}}_{\mathbf{y}}-\mathbf{\Sigma}\right) \mathbf{a}_{k}}{\left\|\mathbf{a}_{k}\right\|_{2}^{4}},-\gamma_{k}\right\}$.

8: $\quad$ Update $\gamma_{k} \leftarrow \gamma_{k}+d^{*}$.

9: Update $\boldsymbol{\Sigma}^{-1} \leftarrow \boldsymbol{\Sigma}^{-1}-\frac{d^{*} \boldsymbol{\Sigma}^{-1} \mathbf{a}_{k} \mathbf{a}_{k}^{H} \boldsymbol{\Sigma}^{-1}}{1+d^{*} \mathbf{a}_{k}^{H} \boldsymbol{\Sigma}^{-1} \mathbf{a}_{k}}$

10: end for

11: Output: The resulting estimate $\gamma$.

\section{Iterative Algorithm}

The presented estimators can both be found using an iterative componentwise minimization algorithm: Starting from an initial point $\gamma$, at each step of the algorithm we minimize the objective function $f(\gamma)$ with respect to only one of its coordinates $\gamma_{k}$. Of course, the component update step is different in the case of ML and in the case of NNLS. The derivation of the update rules can be found in [22], we summarize them in Algorithm 1 . Variants of the algorithm may differ in the way the initial point is chosen and in the way the components are chosen for update. We can also include the noise variance $\sigma^{2}$ as an additional optimization parameter and estimate it along $\gamma$.

\section{Asymptotic scaling}

In this section we discuss the performance of the NNLS estimator in a single slot $(S=1)$. For the sake of simplicity, in the discussion of this section we assume $g_{k}=1$ for all $k$. In this case, the SNR $E_{s} / N_{0}$ is also the SNR at the receiver, for each individual (active) user and $\|\gamma\|_{1} / \sigma^{2}=K_{a} E_{s} / N_{0}$. It is shown in [9. Corollary 2] that

$$
\frac{\left\|\gamma-\gamma^{*}\right\|_{1}}{\|\gamma\|_{1}} \leq c\left(1+\left(K_{a} \frac{E_{s}}{N_{0}}\right)^{-1}\right) \sqrt{\frac{K_{a}}{M}}
$$

holds with high probability provided that

$$
K_{a}=\mathcal{O}\left(L^{2} / \log ^{2}\left(e 2^{J} / L^{2}\right)\right)
$$

where $c>0$ is some universal constant and $\gamma^{*}$ denotes the estimate of $\gamma$ by the NNLS algorithm (see section III-B). Numerical results [22] suggest that the reconstruction error of the ML algorithm is at least as good as that of NNLS (in practice it is much better). This bound is indeed very conservative. Nevertheless, this is enough to give achievable scaling laws for the probability of error of the decoder. It follows from (15) that $\frac{\left\|\boldsymbol{\gamma}-\boldsymbol{\gamma}^{*}\right\|_{1}}{\|\boldsymbol{\gamma}\|_{1}} \rightarrow 0$ for $\left(M, K_{a}, \frac{E_{s}}{N_{0}}\right) \rightarrow(\infty, \infty, 0)$ as long as

$$
\frac{K_{a}\left(1+\left(K_{a} E_{s} / N_{0}\right)^{-1}\right)^{2}}{M}=o(1),
$$


which is satisfied if $M$ grows as

$$
M=\max \left(K_{a},\left(E_{s} / N_{0}\right)^{-1}\right)^{\kappa}
$$

for some $\kappa>1$. Assuming that $J$ scales such that $2^{J}=\delta L^{2}$ for some fixed $\delta \geq 1$, i.e. $J=\mathcal{O}(\log L)$, then the scaling condition (16) becomes $K_{a}=\mathcal{O}\left(L^{2}\right)$ and we can conclude that the recovery error vanishes for sum spectral efficiencies up to

$$
\frac{K_{a} J}{L}=\mathcal{O}(L \log L)
$$

This shows that, in principles, we can achieve a total spectral efficiency that grows without bound, by encoding over larger and larger blocks of dimension $L$, as long as the number of messages per user and the number of active users both grow proportionally to $L^{2}$, when the number of BS antennas scales as in (18), this system achieves a sum spectral efficiency that grows with $L \log (L)$ and an error probability as small as desired, for any given $E_{b} / N_{0}>0$. Of course, in this regime the rate per active user vanishes as $\log (L) / L$.

\section{REDUCING COMPLEXITY VIA CONCATENATED CODING}

In practice it is not feasible to transmit even small messages (e.g. $J \sim 100)$ within one coherence block $(S=1)$, because the number of columns in the coding matrix $\mathbf{A}$ grows exponentially in $J$. Let each user transmit his message over a frame of $S$ fading blocks and within each block use the code described in section $\amalg$ as inner code with the ML decoder as inner decoder.

We follow the concatenated coding scheme approach of [8], suitably adapted to our case. Let $B=n R$ denote the number of bits per user message. For some suitable integers $S \geq 1$ and $J>0$, we divide the $B$-bit message into blocks of size $b_{1}, b_{2}, \ldots, b_{S}$ such that $\sum_{s} b_{s}=B$ and such that $b_{1}=J$ and $b_{s}<J$ for all $s=2, \ldots, S$. Each subblock $s=2,3, \ldots, S$ is augmented to size $J$ by appending $p_{s}=J-b_{s}$ parity bits, obtained using pseudo-random linear combinations of the information bits of the previous blocks $s^{\prime}<s$. Therefore, there is a one-to-one association between the set of all sequences of coded blocks and the paths of a tree of depth $S$. The pseudorandom parity-check equations generating the parity bits are identical for all users, i.e., each user makes use exactly of the same outer tree code. For more details on the outer coding scheme, please refer to [8].

Given $J$ and the slot length $L$, the inner code is used to transmit in sequence the $S$ (outer-encoded) blocks forming a frame. Let $\mathbf{A}$ be the coding matrix as defined in section II Each column of $\mathbf{A}$ now represents one inner codeword. Letting $i_{k}(1), \ldots, i_{k}(S)$ denote the sequence of $S$ (outer-)encoded $J$ bit messages produced by the outer encoder of active user $k \in \mathcal{K}_{a}$. The user $k$ now simply sends in sequence, over consecutive slots of length $L$, the columns $\mathbf{a}_{i_{k}(1)}, \mathbf{a}_{i_{k}(2)}, \ldots, \mathbf{a}_{i_{k}(S)}$ of the coding matrix $\mathbf{A}$. As described in section II the inner decoding problem is equivalent to the AD problem (5). For each subslot $s$, let $\widehat{\gamma}[s]=\left(\widehat{\gamma}_{1}[s], \ldots, \widehat{\gamma}_{2^{J}}[s]\right)^{\top}$ denote the ML estimate of $\gamma$ in subslot $s$ obtained by the inner decoder. Then, the list of active messages at subslot $s$ is defined as

$$
\mathcal{S}_{s}=\left\{r \in\left[2^{J}\right]: \widehat{\gamma}_{r}[s] \geq \nu_{s}\right\}
$$

where $\nu_{1}, \ldots, \nu_{S}$ are suitable pre-defined thresholds. Let $\mathcal{S}_{1}, \mathcal{S}_{2}, \ldots, \mathcal{S}_{S}$ the sequence of lists of active subblock messages. Since the subblocks contain parity bits with parity profile $\left\{0, p_{2}, \ldots, p_{S}\right\}$, not all message sequences in $\mathcal{S}_{1} \times \mathcal{S}_{2} \times$ $\cdots \times \mathcal{S}_{S}$ are possible. The role of the outer decoder is to identify all possible message sequences, i.e., those corresponding to paths in the tree of the outer tree code [8]. The output list $\mathcal{L}$ is initialized as an empty list. Starting from $s=1$ and proceeding in order, the decoder converts the integer indices $\mathcal{S}_{s}$ back to their binary representation, separates data and parity bits, computes the parity checks for all the combinations with messages from the list $\mathcal{L}$ and extends only the paths in the tree which fulfill the parity checks. A precise analysis of the error probability of such a decoder and its complexity in terms of surviving paths in the list is given in [8].

The performance of the concatenated system is demonstrated via simulations in section $\mathrm{V}$

\section{A. Asymptotic analysis - Outer code}

We define the support $\boldsymbol{\rho}[s]$ of the estimated $\widehat{\gamma}[s]$ as a binary vector whose $r$-th element is equal to 1 if $\widehat{\gamma}_{r}[s] \geq \nu_{s}$ and to zero otherwise. In the case of error-free support recovery, $\boldsymbol{\rho}[s]$ can be interpreted as the output of a vector "OR" multiple access channel (OR-MAC) where the inputs are the binary columns of the activity matrix $\boldsymbol{\Phi}[s]$ and the output is given by

$$
\boldsymbol{\rho}[s]=\bigvee_{k \in \mathcal{K}_{a}} \boldsymbol{\Phi}_{:, k}[s],
$$

where $\bigvee$ denotes the component-wise binary OR operation. The logical "OR" arises from the fact that if the same submessage is selected by multiple users, it will shows up as "active" at the output of the "activity-detection" inner decoder since the signal energy adds up (as discussed before).

\section{1) Achievability}

The analysis in [8] shows that the error probability of the outer code goes to zero in the so called logarithmic regime with constant outer rate, i.e. for $K_{a}, J \rightarrow \infty$ as $J=\alpha \log _{2} K_{a}$ and $B=S R_{\text {out }} J{ }^{4}$ if the number of parity bits $P$ is chosen as $([8$, Theorem 5 and 6])

1) $P=(S+\delta-1) \log _{2} K_{a}$ for some constant $\delta>0$ if all the parity bits are allocated in the last slots.

2) $P=c(S-1) \log _{2} K_{a}$ for some constant $c>1$ if the parity bits are allocated evenly at the end of each subslot except for the first.

In the first case the complexity scales like $\mathcal{O}\left(K_{a}^{R_{\text {out }} S} \log K_{a}\right)$, since there is no pruning in the first $R_{\text {out }} S$ subslots, while in the second case the complexity scales linearly with $S$ like

\footnotetext{
${ }^{4}$ We deviate slightly from the notation in [8], where the scaling parameter $\alpha^{\prime}$ is defined by $B=\alpha^{\prime} \log _{2} K_{a}$ and the number of subslots is considered to be constant. It is apparent that those definitions are connected by $\alpha^{\prime}=S R_{\text {out }} \alpha$.
} 
$\mathcal{O}\left(S K_{a} \log K_{a}\right)$. The corresponding outer rates are

$$
\begin{aligned}
R_{\mathrm{out}} & =B /(B+P) \\
& =1-P /(B+P) \\
& =1-P /(S J) \\
& =1-\frac{S+\delta-1}{S \alpha} \\
& =1-\frac{1}{\alpha}+\frac{1}{S} \frac{\delta-1}{\alpha}
\end{aligned}
$$

for the case of all parity bits in the last sections and

$$
\begin{aligned}
R_{\text {out }} & =1-\frac{c(S-1)}{S \alpha} \\
& =1-\frac{c}{\alpha}-\frac{c}{S \alpha}
\end{aligned}
$$

for the case of equally distributed parity bits. In the limit $S \rightarrow$ $\infty$ the achievable rates are therefore $R_{\text {out }}=1-1 / \alpha$ and $R_{\text {out }}=1-c / \alpha$ respectively.

\section{2) Converse}

The output entropy of the vector OR-MAC of dimension $2^{J}$ is bounded by the entropy of $2^{J}$ scalar OR-MACs. The marginal distribution of the entries of $\boldsymbol{\rho}[s]$ is Bernoulli with $\mathbb{P}\left(\rho_{r}[s]=0\right)=\left(1-2^{-J}\right)^{K_{a}}$. Hence, we have

$$
H(\rho[s]) \leq 2^{J} \mathcal{H}_{2}\left(\left(1-2^{-J}\right)^{K_{a}}\right) .
$$

We stay in the logarithmic scaling regime, introduced in the previous sections, i.e. we fix $J=\alpha \log _{2} K_{a}$ for some $\alpha>1$ and consider the limit $K_{a}, J \rightarrow \infty$. In this regime $K_{a} / 2^{J}=$ $K_{a}^{-(\alpha-1)} \rightarrow 0$ and we have $1-\left(1-2^{-J}\right)^{K_{a}}=K_{a} / 2^{J}+$ $\mathcal{O}\left(\left(K_{a} / 2^{J}\right)^{2}\right) \rightarrow 0$. This gives that

$2^{J} \mathcal{H}_{2}\left(\left(1-2^{-J}\right)^{K_{a}}\right) \rightarrow K_{a}\left(J-\log _{2} K_{a}\right)=(\alpha-1) K_{a} \log _{2} K_{a}$.

Since all users make use of the same code we have that the number of information bits sent by the $K_{a}$ active users over a slot is $B_{\text {sum }}=K_{a} J R_{\text {out }}$. Therefore, in order to hope for small probability of error a necessary condition is

$$
K_{a} J R_{\text {out }} \leq 2^{J} \mathcal{H}_{2}\left(\left(1-1 / 2^{J}\right)^{K_{a}}\right) .
$$

So the outer rate is limited by

$$
R_{\text {out }} \leq(\alpha-1) \frac{\log _{2} K_{a}}{J}=1-\frac{1}{\alpha} .
$$

We have shown in the previous section that this outer rate can be achieved in the limit of infinite subslots $S \rightarrow \infty$ by the described outer tree code at the cost of a decoding complexity of at least $\mathcal{O}\left(K_{a}^{R_{\text {out }} S}\right)$ or up to a constant factor $\Delta R_{\text {out }}=(c-1) / \alpha$ for some $c>1$ with a complexity of $\mathcal{O}\left(S K_{a} \log K_{a}\right)$. This is a noteworthy results on its own, since it is a priori not clear, whether the bound 26 is achievable by an unsourced random access scheme, i.e. each user using the same codebook.

The resulting achievable sum spectral efficiency can be calculated as in section III-D with a subtle but important difference, since the results on the outer code are valid only in the logarithmic regime $J=\alpha \log _{2} K_{a}$, i.e. $2^{J}=K_{a}^{\alpha}$ for $\alpha>1$. According to [22, Corollary 2] the error probability of the inner code vanishes if the number of active users scale no faster then $K_{a}=\mathcal{O}\left(L^{2} / \log ^{2}\left(e 2^{J} / L^{2}\right)\right)$. Using the scaling condition $J=\alpha \log _{2} K_{a}$ and that $K_{a} \leq L^{2}$, this implies that in the logarithmic regime the error probability of the inner code vanishes if the number of active users scales as $K_{a}=\mathcal{O}\left(L^{2} / \log ^{2}(L)\right)$. This gives a sum spectral efficiency of

$$
\frac{K_{a} R_{\mathrm{out}} J}{L}=\mathcal{O}\left(\frac{K_{a} \log K_{a}}{L}\right)=\mathcal{O}\left(\frac{L}{\log L}\right) .
$$

The order of this sum spectral efficiency is, by a factor $\log ^{2} L$, smaller then the one we calculated in section III-D This is because the order of supported active users is smaller by exactly the same $\log ^{2} L$ factor. In section III-D we assumed that $J$ scales as $2^{J}=\delta L^{2}=\mathcal{O}\left(K_{a}\right)$ for some $\delta>1$, so that the ratio $K_{a} / 2^{J}$ remains constant. It is not clear from the analysis in [8], whether the probability of error of the outer tree code would vanish in the regime. We can get a converse by evaluating the entropy bound (26). Let $2^{J}=\delta K_{a}$ with $\delta>1$, then $\left(1-2^{-J}\right)^{K_{a}}=\left(1-\delta / K_{a}\right)^{K_{a}} \underset{K_{a} \rightarrow \infty}{\longrightarrow} \exp (-\delta)$. Therefore the binary entropy $\mathcal{H}_{2}\left(\left(1-2^{-J}\right)^{K_{a}}\right)$ remains a constant in the limit $J, K_{a} \rightarrow \infty$ and we get that

$$
K_{a} R_{\text {out }} J \leq \delta K_{a} \mathcal{H}_{2}(\exp (-\delta)) .
$$

This shows that $R_{\text {out }} \rightarrow 0$ in the limit $J, K_{a} \rightarrow \infty$ is the best achievable asymptotic per-user outer rate, but the outer sum rate $K_{a} R_{\text {out }} J$ is proportional to $K_{a}$. The resulting sum spectral efficiencies scale as

$$
\frac{K_{a} R_{\text {out }} J}{L}=\mathcal{O}\left(\frac{K_{a}}{L}\right)=\mathcal{O}(L) .
$$

This means it could be possible to increase the achievable sum spectral efficiencies by a factor of $\log L$ by using an outer code that is able to achieve the entropy bound $\sqrt{26}$ in the regime $2^{J}=\delta K_{a}$. It is not clear though whether the code of [8] or some other code can achieve this.

\section{Simulations}

The outer decoder requires a hard decision on the support of the estimated $\widehat{\gamma}[s]$. When $K_{a}$ is known, one approach consists of selecting the $K_{a}+\Delta$ largest entries in each section, where $\Delta \geq 0$ can be adjusted to balance between false alarm and misdetection in the outer channel. However, the knowledge of $K_{a}$ is a very restrictive assumption in such type of systems. An alternative approach, which does not require this knowledge, consists of fixing a sequence of thresholds $\left\{\nu_{s}: s \in[S]\right\}$ and let $\boldsymbol{\rho}[s]$ to be the binary vector of dimension $2^{J}$ with elements equal to 1 for all components of $\widehat{\gamma}[s]$ above threshold $\nu_{s}$. By choosing the thresholds, we can balance between missed detections and false alarms. Furthermore, we may consider the use of a non-uniform decaying power allocation across the slots as described in [9].

For the simulations in Figure 1 we choose $B=96$ bits as payload size for each user, a frame of choose $S=32$ slots of $L=100$ dimensions per slot, yielding an overall block length $n=3200$. Choosing the binary subblock length $J=12$, the inner coding matrix A has dimension $100 \times 4096$ and therefore is still quite manageable. We choose the columns of 


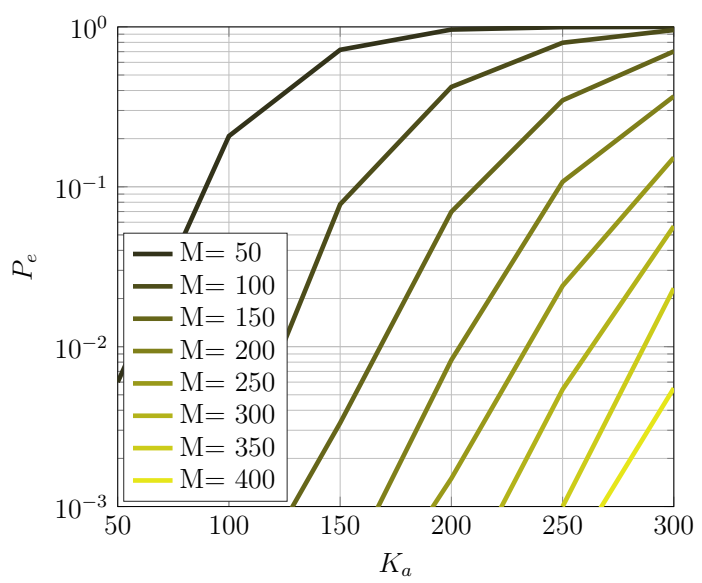

Fig. 1: Error probability $\left(P_{e}=p_{\mathrm{md}}+p_{\mathrm{fa}}\right)$ as a function of the number of active users for different numbers of receive antennas. $E_{b} / N_{0}=0 \mathrm{~dB}, L=100, n=3200, b=96$ bits, $S=32, J=12$.

A uniformly i.i.d. from the sphere of radius $\sqrt{L}$. Notice also that if one wishes to send the same payload message using the piggybacking scheme of [26, 33], each user should make use of $2^{96}$ columns, which is totally impractical.

For the outer code, we choose the following parity profile $p=[0,9,9, \ldots, 9,12,12,12]$, yielding an outer coding rate $R_{\text {out }}=0.25$ information bits per binary symbol. All large scale fading coefficients are fixed to $g_{k} \equiv 1$. In Figure 1 we fix $N_{0}=1$ and choose the transmit power (energy per symbol), such that $E_{b} / N_{0}=0 \mathrm{~dB}$ and plot the sum of the two types of error probabilities $P_{e}=p_{\mathrm{md}}+p_{\mathrm{fa}}$ (see (1) and (2)) as a function of the number of active users for different numbers of receive antennas $M$. Figure 2 shows how $P_{e}$ falls as a function of $E_{b} / N_{0}$ for different values of $K_{a}$ and $M$. Table I summarizes the required values of $E_{b} / N_{0}$ to achieve a total error probability $P_{e}<0.05$. Notice that this corresponds to a total spectral efficiency $\mu=\frac{12}{100} \times 0.25 \times 300=9$ bit per channel use, which is significantly larger than today's LTE cellular systems (in terms of bit/s/Hz per sector) and definitely much larger than IoT-driven schemes such as LoRA [34, 35]. According to the random coding bound of [5] this is impossible to achieve for the scalar Gaussian channel (only one receive antenna), even with coherent detection and roughly five times smaller spectral efficiency then here. This shows also quantitatively that the non-coherent massive MIMO channel is very attractive for U-RA, since it preserves the same attractive characteristics of U-RA as in the non-fading Gaussian model of [5] (users transmit without any pre-negotiation, and no use of pilot symbols is needed), while the total spectral efficiency can be made as large as desired simply by increasing the number of receiver antennas.

\section{CONCLUSION}

In this paper we studied the problem of unsourced random access, a special type of grant-free random access, for the block-fading channel with a massive MIMO BS. We showed that an arbitrarily fixed probability of error can be achieved

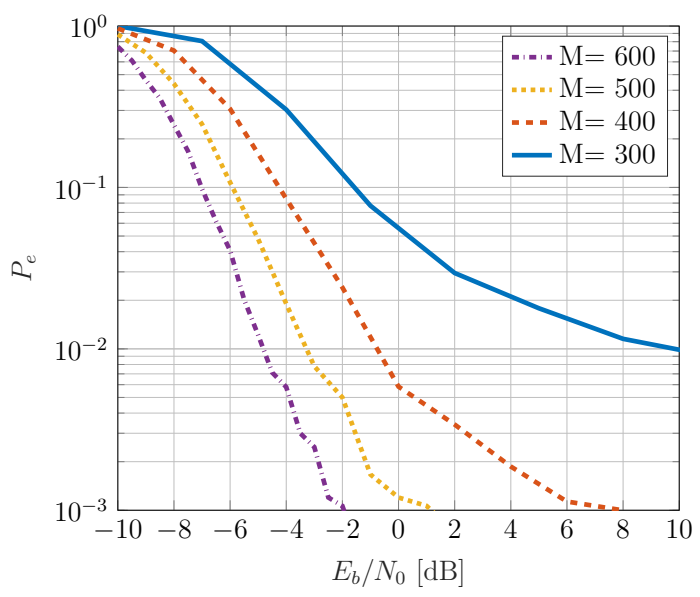

(a) $K_{a}=300$

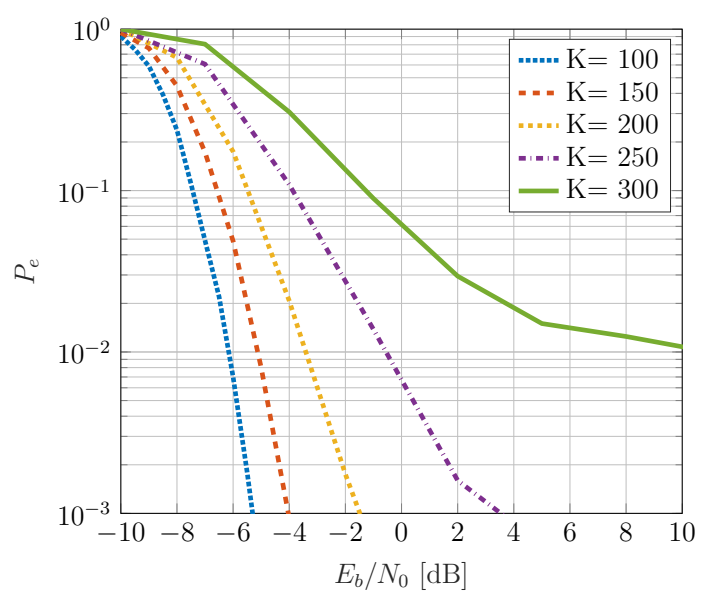

(b) $M=300$

Fig. 2: Error probability $\left(P_{e}=p_{\mathrm{md}}+p_{\mathrm{fa}}\right)$ as a function of $E_{b} / N_{0} . L=100, n=3200, b=96$ bits, $S=32, J=12$.

\begin{tabular}{|c|c|c|c|c|}
\hline$M$ & 300 & 400 & 500 & 600 \\
\hline$E_{b} / N_{0}[\mathrm{~dB}]$ & 0.4 & -3.1 & -5.0 & -6.2 \\
\hline
\end{tabular}

(a) $K_{a}=300$

\begin{tabular}{|c|c|c|c|c|c|}
\hline$K_{a}$ & 100 & 150 & 200 & 250 & 300 \\
\hline$E_{b} / N_{0}[\mathrm{~dB}]$ & -7.0 & -6.0 & -4.8 & -2.9 & 0.6 \\
\hline
\end{tabular}

(b) $M=300$

TABLE I: Required $E_{b} / N_{0}$ to achieve a total error probability $P_{e}<0.05$ with $L=100, n=3200, b=96$ bits, $S=32, J=$ 12.

at any $E_{b} / N_{0}$ for sufficiently large number of antennas, and a total spectral efficiency that grows as $O(L \log L)$, where $L$ is the code block length, can be achieved. Such oneshot scheme is conceptually nice but not suited for typical practical applications with message payload of the order of $B \approx 100$ bits, since it would require a codebook matrix with $2^{B}$ columns. Hence, we have also considered the application of the concatenated approach pioneered in [8], where the message is broken into a sequence of smaller blocks and the activity detection scheme is applied as an inner encoding/decoding stage at each block, while an outer tree code takes care of "stitching together" the sequence of decoded submessages over 
the blocks. We show that the concatenated coding scheme can achieve a total spectral efficiency of $\mathcal{O}(L / \log L)$. Numerical simulations show the effectiveness of the proposed method. It should be noticed that these schemes are completely noncoherent, i.e., the receiver never tries to estimate the massive MIMO channel matrix of complex fading coefficients. Therefore, the scheme pays no hidden penalty in terms of pilot symbol overhead, often connected with the assumption of ideal coherent reception, i.e., channel state information known to the receiver.

\section{ACKNOWLEDGEMENT}

P.J. is supported by DFG grant JU 2795/3.

\section{REFERENCES}

[1] T. Taleb and A. Kunz, "Machine type communications in 3GPP networks: Potential, challenges, and solutions," IEEE Commun. Mag., vol. 50, no. 3, pp. 178-184, Mar. 2012.

[2] M. Hasan, E. Hossain, and D. Niyato, "Random access for machine-to-machine communication in LTE-advanced networks: Issues and approaches," IEEE Commun. Mag., vol. 51, no. 6, pp. 86-93, Jun. 2013.

[3] S. Sesia, I. Toufik, and M. Baker, LTE, The UMTS Long Term Evolution: From Theory to Practice. Wiley Publishing, 2009.

[4] M. Agiwal, A. Roy, and N. Saxena, "Next Generation 5G Wireless Networks: A Comprehensive Survey," IEEE Commun. Surv. Tutor., vol. 18, no. 3, pp. 1617-1655, 2016.

[5] Y. Polyanskiy, "A perspective on massive random-access," in 2017 IEEE International Symposium on Information Theory (ISIT), Jun. 2017, pp. 2523-2527.

[6] O. Ordentlich and Y. Polyanskiy, "Low Complexity Schemes for the Random Access Gaussian Channel," 2017 IEEE Int. Symp. Inf. Theory ISIT, pp. 2533-2537, 2017.

[7] R. Calderbank and A. Thompson, "CHIRRUP: a practical algorithm for unsourced multiple access," arXiv:1811.00879, Nov. 2018.

[8] V. K. Amalladinne, A. Vem, D. K. Soma, K. R. Narayanan, and J.-F. Chamberland, "A Coupled Compressive Sensing Scheme for Uncoordinated Multiple Access," arXiv:1809.04745, Sep. 2018.

[9] A. Fengler, P. Jung, and G. Caire, "SPARCs and AMP for Unsourced Random Access," in IEEE International Symposium on Information Theory (ISIT), Jul. 2019, pp. 2843-2847.

[10] V. K. Amalladinne, J.-F. Chamberland, and K. R. Narayanan, "An enhanced decoding algorithm for coded compressed sensing," arXiv:1910.09704, Oct. 2019.

[11] E. Marshakov, G. Balitskiy, K. Andreev, and A. Frolov, "A Polar Code Based Unsourced Random Access for the Gaussian MAC," in 2019 IEEE 90th Vehicular Technology Conference (VTC2019Fall), Sep. 2019, pp. 1-5.

[12] A. K. Pradhan, V. K. Amalladinne, K. R. Narayanan, and J.F. Chamberland, "Polar Coding and Random Spreading for Unsourced Multiple Access," arXiv:1911.01009, Nov. 2019.

[13] S. S. Kowshik, K. Andreev, A. Frolov, and Y. Polyanskiy, "Energy efficient random access for the quasi-static fading MAC," in 2019 IEEE International Symposium on Information Theory (ISIT). Paris, France: IEEE, Jul. 2019, pp. 2768-2772.

[14] D. Tse and P. Viswanath, Fundamentals of wireless communication. Cambridge University Press, 2005.

[15] J. Chen and X. Huo, "Theoretical results on sparse representations of multiple-measurement vectors," IEEE Trans Signal Process, pp. 4634-4643, 2006.
[16] S. Cotter, B. Rao, Kjersti Engan, and K. Kreutz-Delgado, "Sparse solutions to linear inverse problems with multiple measurement vectors," IEEE Trans. Signal Process., vol. 53, no. 7, pp. 2477-2488, Jul. 2005.

[17] J. M. Kim, O. K. Lee, S. Member, and J. C. Ye, "Compressive MUSIC: Revisiting the link between compressive sensing and array signal processing," IEEE Trans Inf. Theory, pp. 278-301, 2012.

[18] S. Haghighatshoar, P. Jung, and G. Caire, "Improved scaling law for activity detection in massive mimo systems," in 2018 IEEE International Symposium on Information Theory (ISIT), 2018.

[19] P. Pal and P. P. Vaidyanathan, "Pushing the Limits of Sparse Support Recovery Using Correlation Information," IEEE Trans. Signal Process., vol. 63, no. 3, pp. 711-726, Feb. 2015.

[20] C. G. Khatri and C. R. Rao, "Solutions to Some Functional Equations and Their Applications to Characterization of Probability Distributions," Sankhy: The Indian Journal of Statistics, Series A (1961-2002), vol. 30, no. 2, pp. 167-180, 1968.

[21] A. Fengler and P. Jung, "On the Restricted Isometry Property of Centered Self Khatri-Rao Products," arXiv:1905.09245, May 2019.

[22] A. Fengler, S. Haghighatshoar, P. Jung, and G. Caire, "NonBayesian Activity Detection, Large-Scale Fading Coefficient Estimation, and Unsourced Random Access with a Massive MIMO Receiver," arXiv:1910.11266, Oct. 2019.

[23] S. Khanna and C. R. Murthy, "On the Support Recovery of Jointly Sparse Gaussian Sources using Sparse Bayesian Learning," arXiv:1703.04930, Mar. 2017.

[24] _ _ "Corrections to "On the Restricted Isometry of the Columnwise Khatri-Rao Product"," IEEE Trans. Signal Process., vol. 67, no. 9, pp. 2387-2388, May 2019.

[25] Z. Chen and W. Yu, "Phase transition analysis for covariance based massive random access with massive MIMO," in 2019 Asilomar Conference on Signals, Systems, and Computers, 2019.

[26] K. Senel and E. G. Larsson, "Device Activity and Embedded Information Bit Detection Using AMP in Massive MIMO," in 2017 IEEE Globecom Workshops (GC Wkshps), Dec. 2017.

[27] L. Liu and W. Yu, "Massive Connectivity With Massive MIMO - Part I: Device Activity Detection and Channel Estimation," IEEE Transactions on Signal Processing, vol. 66, no. 11, pp. 2933-2946, June 2018.

[28] Z. Chen, F. Sohrabi, and W. Yu, "Sparse Activity Detection for Massive Connectivity," IEEE Trans. Signal Process., vol. 66, no. 7, pp. 1890-1904, Apr. 2018.

[29] T. L. Marzetta and H. Yang, Fundamentals of Massive MIMO. Cambridge University Press, Nov. 2016.

[30] C. Wang, O. Y. Bursalioglu, H. Papadopoulos, and G. Caire, "On-the-fly large-scale channel-gain estimation for massive antenna-array base stations," in 2016 IEEE International Conference on Communications (ICC), 2018.

[31] M. Slawski and M. Hein, "Non-negative least squares for highdimensional linear models: Consistency and sparse recovery without regularization," Electronic Journal of Statistics, vol. 7 , pp. 3004-3056, 2013.

[32] R. Kueng and P. Jung, "Robust Nonnegative Sparse Recovery and the Nullspace Property of 0/1 Measurements," IEEE Trans. Inf. Theory, vol. 64, pp. 689-703, 2017.

[33] E. G. Larsson and R. Moosavi, "Piggybacking an Additional Lonely Bit on Linearly Coded Payload Data," IEEE Wirel. Commun. Lett., vol. 1, no. 4, pp. 292-295, Aug. 2012.

[34] M. Centenaro, L. Vangelista, A. Zanella, and M. Zorzi, "Longrange communications in unlicensed bands: The rising stars in the IoT and smart city scenarios," IEEE Wirel. Commun., vol. 23, no. 5, pp. 60-67, Oct. 2016.

[35] D. Bankov, E. Khorov, and A. Lyakhov, "On the Limits of LoRaWAN Channel Access," in 2016 International Conference on Engineering and Telecommunication (EnT), Nov. 2016, pp. $10-14$. 